Voix et Images

voixetimages

\title{
La littérature québécoise, une littérature du tiers-monde?
}

\section{Gilles Thérien}

Volume 12, numéro 1 (34), automne 1986

Québec-Amérique latine

URI : https://id.erudit.org/iderudit/200602ar

DOI : https://doi.org/10.7202/200602ar

Aller au sommaire du numéro

Éditeur(s)

Université du Québec à Montréal

ISSN

0318-9201 (imprimé)

1705-933X (numérique)

Découvrir la revue

Citer cet article

Thérien, G. (1986). La littérature québécoise, une littérature du tiers-monde?

Voix et Images, 12(1), 12-20. https://doi.org/10.7202/200602ar d'utilisation que vous pouvez consulter en ligne.

https://apropos.erudit.org/fr/usagers/politique-dutilisation/ 


\title{
La littérature québécoise, une littérature du tiers-monde?
}

\author{
par Gilles Thérien, Université du Québec à Montréal
}

\section{Le choc}

Un éminent professeur américain offre un cours de «littérature du tiersmonde". S'y succèdent des ouvres d'auteurs latino-américains, africains, égyptiens, pakistanais, chinois et Une saison dans la vie d'Emmanuel de Marie-Claire Blais. Cette mise en place de notre littérature me fait frémir. Rien contre Marie-Claire Blais découverte par Edmund Wilson et accessible en anglais. Non, ce qui me hante, c'est plutôt la république de bananes, les petites dictatures aux uniformes rutilants, les discours sur le développement et le retard, l'axe nord-sud, les œuvres condamnées à la traduction et à la bienfaisance. J'ai quand même raison de refuser ces fantasmes: le Canada fait partie du groupe des sept pays industrialisés, le gouvernement québécois est toujours d'une légitimité à toute épreuve et quand il a un doute là-dessus, il va aux urnes. Ma culture est fille de la française et ici, je me sens quand même plus européen que les gastronomes du Macdonald. Je me sens soudainement lésé. C'est un cours entier sur la littérature québécoise qu'il faudrait. N'avons-nous pas fait des efforts considérables pour convaincre le monde universitaire entier de nous consacrer ne serait-ce qu'une petite unité d'enseignement et voilà qu'en guise de récompense, nous sommes réduits à la portion congrue et tiersmondialisée. Injustice, trahison? Mais alors les Africains eux? On dira qu'ils sont habitués à pareille réduction et qu'au surplus, c'est la réalité: ils sont pauvres, ce sont d'anciennes colonies, les écrivains doivent choisir entre un dialecte quelconque et la langue du colonisateur... Mais les Chinois dont la civilisation est une des plus anciennes? Ils ont une longue tradition féodale, ils sont fermés sur eux-mêmes et la preuve, c'est qu'il n'est pas question des Japonais! Les Latino-américains fractionnés en pays multiples, victimes de dictatures militaires et d'oppression de toutes sortes? Ça commence à se compliquer, quelques noms d'écrivains me gênent... ils sont du tiers monde, ils le disent, ils le proclament, ils s'en servent... Alors le doute s'installe. Et si c'était vrai? Si notre littérature n'était qu'une littérature du tiers monde, qu'est-ce que cela changerait à notre perception?

Une des principales difficultés à accepter pareille idée tient à ce que nous sommes: un immense pays au nord des États-Unis, dont les deux nations fondatrices se réclament de deux des grandes cultures occidentales, la française et l'anglaise. C'est cela qui constitue notre identité nationale quand on examine la question du point de vue théorique qu'est le Canada, c'est-à-dire du point de vue de ce qui est moins un pays qu'une association. L'autre difficulté tient à la situation particulière du Québec qui, selon les occasions, 
peut jouer alternativement la carte européenne en affirmant sa francité ou la carte nord-américaine au nom de son américanité: le Québec, la plaque tournante entre les deux grandes cultures et les deux grandes langues de l'Occident capitaliste! De quoi donner le vertige.

Les pays du tiers monde sont bien différents. Ils ont une histoire précise: d'une part un colonialisme venu de l'extérieur, ou encore dans certains cas, un féodalisme imposé par une oligarchie, d'autre part une révolution, habituellement sanglante et la prise de pouvoir par de nouvelles élites à qui incombe l'obligation de transformer un pays sous-développé en pays développé. Une conscience collective accompagne ces pays qui se retrouvent dans une organisation comme celle des états américains où le Canada ne siège pas à titre de membre et aussi celle des pays non alignés dont l'objectif est de produire un nouvel ordre qui modifierait l'ordre des deux grands centres actuels, les USA et l'URSS. Les pays du tiers monde expriment collectivement leurs volontés de faire varier les tensions entre les centres et de jouer à plein les périphéries. Le Canada n'èst pas non plus de ce groupe, ni le Québec. La raison en est, croyons-nous, que nous avons le fantasme du centre et pas celui de la périphérie... alors que de toute évidence, nous constituons probablement un cas à part dans l'histoire géopolitique mondiale en réussissant à être, sans même devoir nous en rendre compte, un pays du tiers monde industrialisé et arrivé à cet état par la grâce de ses voisins et sans qu'aucune volonté nationale, tant au Canada' qu'au Québec, n'y soit pour quelque chose: un masque industrialisé sur une âme de colonisé. Si cela est, qu'en est-il du statut de notre littérature, de la littérature québécoise? Vit-elle du même fantasme?

\section{L'existence du fait littéraire québécois}

Au cours des années, nous' nous sommes dotés d'une institution littéraire complète: elle comprend l'enseignement de la littérature à tous les niveaux avec manuels d'histoire littéraire et archives, la publication de livres, de revues et de journaux. On y retrouve une académie, des sociétés d'auteurs, des syndicats d'écrivains, des subventions multiples, des prix littéraires et des jurys, des maisons d'édition, des agences de diffusion, des librairies, - et même des librairies de livres usagés, - une critique universitaire et une critique journalistique. Congrès et colloques se penchent sur notre littérature pour en faire l'examen; cela avec une périodicité effarante. La littérature québécoise est acheminée à l'étranger quand l'étranger n'est pas acheminé ici. Les réseaux intellocratiques se sont développés avec assurance et efficacité depuis les années soixante. Nous avons nos gloires et nos catastrophes [ littéraires ] nationales, notre langue autochtone avec son dictionnaire, nos chapelles littéraires, nos excommunications, nos éternels débats sur la qualité de la langue et la sauvegarde du français, notre désir d'écrire un peu comme à New York et surtout comme à Paris, nos rencontres d'écrivains, nos maisons de la culture, nos exilés, nos rapatriés, nos soirées de débats et nos nuits de poésie. N'est-ce pas ce que l'on retrouve en France suivant une proportionnelle acceptable? En somme, nous avons ici l'infrastructure complète d'un grand centre de culture. Cela suffit-il à faire de nous cette nation française d'Amérique qui traite d'égal à égal avec la métropole parisienne? Le fantasme 
littéraire québécois est un fantasme développé dans le sens est-ouest. Il est demeuré dans l'axe de la colonisation. Il est américano-français. L'institution doit nous maintenir dans le statut de deuxième littérature de langue française selon la formule de Gérard Tougas ${ }^{2} \ldots$ ou encore notre objectif doit être d'exprimer l'Amérique dans la tradition littéraire française, entendons "de la France». Il est rarement question d'être français pour les Américains, encore moins pour les latino-américains. Demeure toujours le rêve d'être une sorte de noblesse provinciale d'où le miracle pourrait tirer un quelconque sujet pour l'amener à Paris et le consacrer. Chaque roman qui a le privilège d'être publié en France est un miracle en puissance. Dans toute cette infrastructure orientée pratiquement du seul côté de la France, il manque la traduction, celle qui ouvrirait notre univers d'abord aux cultures américaines et latinoaméricaines... mais encore faudrait-il vraiment vouloir parler selon l'axe nord-sud, prendre conscience de la totalité de l'Amérique, Nouveau Monde d'un pôle à l'autre, terre de conquête et de métissage où l'Europe est venue organiser horizontalement un territoire vertical.

\section{Une thématique tiersmondiste...}

... ou la littérature comme moyen de prendre possession de soi-même. Si depuis la guerre, et plus particulièrement depuis 1960, le roman québécois apparaît de plus en plus comme le moyen privilégié d'expression des écrivains, il faut se demander si cela est surtout dû à la découverte de la puissance du romanesque ou au passage d'une maîtrise de la sémantique visible dans la poésie, à la difficile manipulation de la syntaxe dans un univers où les structures syntaxiques sont plus confuses que les réscaux sémantiques. Savoir nommer la vie en langue québécoise est autre chose que la raconter sur un mode américain ou un mode français. Le roman ne s'écrit pas avec la facilité de la prose de monsieur Jourdain et les tâtonnements de nos romanciers, et de nos meilleurs, pour se trouver un style, une manière, pour passer au creuset de la littérature leurs perceptions quotidiennes, en disent long sur cet apprentissage dont profiteront sans trop se rendre compte les générations futures. Une vision se crée, une norme, - au sens de fixer la barre un peu plus haut, - un imaginaire ${ }^{3}$. Mais il faut compter parmi les balbutiements, les thématiques qui disent le b-a ba de l'entreprise d'écrire. Nous reconnaîtrons aisément, parmi celles-ci, la thématique identitaire, celle du héros qui cherche son identité en même temps qu'il essaie de cerner et de nommer le territoire, et la thématique de l'écrivain.

Dans le premier cas, la thématique identitaire oscille entre deux pôles, celui du sujet qui raconte et se raconte et celui du territoire de son activité, ce que l'on nomme pays mais qui prend presqu'autant de sens qu'il y a de romanciers ${ }^{4}$. Le héros du roman québécois, qu'il s'exprime au «je» ou non, se présente souvent comme le porte-étendard de la collectivité. Les personnages ont de la difficulté à s'individualiser, ils assument comme nature profonde une partie de la société, ils épousent des combats collectifs dans lesquels vient se fondre leur identité, ou encore ils organisent la marge à partir de laquelle ils observent la caravane qui passe. Le territoire, lui, est multiple dans ses acceptions, plus ou moins réel, plus ou moins englobant, il prend 
parfois le devant de la scène et écrase les personnages de sa propre quête identitaire. Il est rarement ce territoire sûr et solide à partir duquel on peut construire, qui ne sert qu'à poser les pieds des personnages. La thématique identitaire se nourrit de l'exploration et de la découverte comme si tout était encore à faire ou à recommencer. Thématique tiersmondiste, puisque, quelque part, le sentiment de dépossession oblige à de nouveaux arpentages, ce pays dont on n'est jamais certain, ce pays qu'il faut construire ou reconstruire, ce coin français en Amérique qu'il faut faire reconnaître dans des accents tout proches du "Vive le Québec libre!». Thématique tiersmondiste que ce héros qui porte sur ses épaules le destin de sa collectivité, de son peuple. Il ne chante pas la victoire, la révolution accomplie; il remet en place les morceaux d'une explosion qui n'a jamais eu lieu. Il ne raconte pas les débuts, il les appelle.

Le thématique de l'écrivain appartient au même ordre de symptômes. Le romancier programme son propre échec par le truchement du romancier fictif qui, lui, n'arrive pas à produire l'œuvre dont il rêves. L'œuvre porte son propre jugement, et parfois sa condamnation. Ce qui aurait pu être un artifice romanesque apparaît souvent comme l'incapacité pour l'auteur de prendre la distance suffisante par rapport à son geste d'écriture. Thématique tiersmondiste que ce double appel à la reconnaissance ou à la condamnation: le livre et l'écrivain seront exaltés ou condamnés ensemble... comme s'il ne fallait pas remettre vingt fois sur le métier, garder au tiroir ou carrément jeter à la poubelle. Tiersmondiste aussi que ce héros scribe tout juste bon à noter les propos du maître-auteur, incapable de prendre seul son droit de parole. Dans l'entreprise de l'écrivain, ce qu'il perçoit comme une impuissance sociale ne parvient pas à se métamorphoser en autre chose qu'en représentation de cette impuissance. La littérature y perd.

Un autre aspect de l'écriture romanesque québécoise engage la réflexion: la dissociation entre la maîtrise de l'écriture et les événements qui marquent notre société depuis la révolution tranquille. Ce qu'apprennent à maîtriser les romanciers, ce sont les techniques du roman réaliste, en particulier l'effet de réel non par une quelconque adéquation entre une référence extérieure et le mot écrit mais par la construction réussie d'un objet littéraire qui sert de mimésis. Au moment où les gouvernements élaborent les diverses nuances de l'autonomie, les frontières du nationalisme, les projets de société qui doivent guider les Québécois, les romanciers sont à plein dans une entreprise qui présuppose l'identité, l'autonomie, le pouvoir et la stabilité de l'État. Ils construisent un objet littéraire qui cherche sa mimésis, qui la programme dans un avenir de science-fiction. La maîtrise de l'effet de réel est un effet du pouvoir. Cela pourrait expliquer le sentiment de schizoïdie croissante entre l'entreprise romanesque et le développement politique de la société. On peut aussi estimer que le retour du pendule en politique n'a pas dérangé les écrivains qui se seront durant tout ce temps donné des moyens de progresser et de mieux contrôler leur écriture. On pourrait, par des études comparatives, reconnaître une même démarche dans les littératures latino-américaines. Les régimes politiques, les bouleversements n'ont pas empêché les écrivains de 
façonner leur art, bien au contraire. Il pourrait s'agir, ici aussi, d'un trait tiersmondiste, quand les écrivains ne s'identifient pas à l'élite au pouvoir mais se chargent d'en être les observateurs méfiants.

Sorte de corollaire de cette thématique tiersmondiste et des expériences d'écriture qu'elle engage, la déconstruction n'apparaît pas viable. L'écriture ne peut se permettre l'expérimentation de la déconstruction que dans la mesure où elle n'est pas plutôt en train d'apprendre à construire. Il est contradictoire de voir les écrivains québécois, confrontés au plan international par une esthétique de la modernité et de la postmodernité et continuer d'écrire leurs proses conservatrices. Cela est encore plus contradictoire quand ces écrivains sont aussi des professeurs qui se targuent d'être, au plan théorique, des véhicules de la modernité. Mais en fait, nous retrouvons ici aussi la contrainte tiersmondiste. Pour se sentir à l'aise dans le courant universel, encore faut-il se sentir à l'aise dans son propre courant, encore faut-il déjà véhiculer des valeurs solides, bien identifiées. La mouvance actuelle ne permet pas de reconnaître une sorte de discours commun, homogène chez les écrivains. La diversité des expériences témoigne plutôt d'un cheminement qui se veut antérieur au coulage dans le béton de quelques idées-forces. La déconstruction devra venir de l'intérieur comme un phénomène naturel.

\section{Le tiersmondisme en études littéraires}

Dans l'institution littéraire, il n'y a pas que l'écriture qui soit piégée. L'enseignement et la recherche le sont aussi. Traditionnellement au Québec, l'enseignement de la littérature est celui de la française à laquelle on ajoute en complément, en appendice, la littérature québécoise. C'est accepter le point de vue de la norme, de ce qui se définit à partir du centre qu'est Paris. La véritable littérature québécoise naît après-guerre. Ce qui précède n'est que balbutiement. Mais la nouvelle littérature en est une de combat, la séparation du Québec, la lutte contre l'américanisation, c'est du moins ce que l'on soupçonne et que l'on cherche continuellement derrière le propos du dernier écrivain: en quoi se rattache-t-il à la grande tradition française, comme le Belge ou le Suisse, ou encore à la grande tradition francophone comme le Maghrébin ou l'Antillais? Longtemps les chercheurs québécois ont épousé les mêmes points de vue. La norme littéraire est prise en France. Du coup on considère que les écrits de la Nouvelle-France sont tout au plus de la prélittérature et qui plus est, à part Madame Bégon, un corpus qui appartient aux Français puisqu'écrit par des Français. De leur côté, les Français ne reconnaissent pas ce corpus comme le leur puisqu'il parle de choses qu'ils ne comprennent pas, de choses qui finalement n'ont pas trait à la France profonde. Le XIX ième siècle canadien-français est présenté comme une véritable entrée en littérature mais sous le mode de l'imitation et de la nostalgie. La première moitié $\mathrm{du} X \mathrm{XX}^{\text {ième }}$ siècle nous rapproche des préoccupations françaises, surtout les chrétiennes, tout en marquant habituellement le retard obligatoire qu'on peut mesurer entre ce qui se passe au centre et ce qui se passe à la périphérie. Depuis les années soixante, le chercheur québécois se sent plus à l'aise pour traiter de la littérature québécoise comme telle. Elle perd suffisamment sa saveur de terroir, son exotisme, pour penser trouver 
une place au sein du discours universel. L'enseignement suit alors les normes françaises. On n'hésite pas au niveau secondaire ou collégial à présenter la liste des "classiques" de notre littérature et, au niveau universitaire, on consacre volontiers des périodes de quarante-cinq heures à des auteurs comme Aquin, Bessette, Hébert ... etc tout comme les Français consacrent quarantecinq heures à Balzac, Flaubert ou Zola. À défaut d'un nouveau roman, on épie les traces de modernité. Force est de constater que les populations étudiantes ont parfois un certain mal à digérer cette immersion. Le comparatisme français avoué, marqué ou simplement inféré finit par appauvrir ce que l'on maintient sur un piédestal. Peut-être n'est-il tout simplement pas possible de parler de la littérature québécoise comme de la française, - ou comme d'une grande littérature, - la richesse des explorations n'étant pas suffisante. C'est encore la même tradition qui nous pousse à diviser, à régionaliser la littérature québécoise dans l'espoir de retrouver une couleur locale suffisamment intéressante pour qu'on la retienne... comme s'il y avait une littérature des provinces françaises... Ici, les études folkloriques et littéraires viennent occuper le même champ.

D'autres aspects de la recherche et de la pratique universitaires sont encore plus subtilement dépendants. Depuis les années soixante, depuis le renouvellement de la théorie littéraire et de la critique, les chercheurs ont eu tendance à calquer leurs réflexions théoriques et leurs analyses sur ce qui se tramait dans les diverses officines françaises, phases thématicienne, symbolique, structuraliste, psychanalytique, marxiste, socio-historique, sémiotique, herméneutique... Nous les avons toutes adoptées et pratiquées. Peu d'essayistes se sont posés des questions sur les transferts idéologiques qu'impliquait l'appropriation de ces divers discours d'école de sorte qu'il est possible que beaucoup de concepts dits opératoires le sont bien peu lorsqu'appliqués à des cultures spécifiques à moins de supposer le caractère universel, achevé et fermé de toutes ces entreprises, à moins de refuser de les lier à l'émergence dans la société européenne de mouvements idéologiques importants qui n'étaient pas de nature esthétique et littéraire.

De plus, il semble difficile de croire que l'état de la théorie en littérature ne corresponde pas à l'état de la littérature elle-même. La polyphonie bakhtinienne, les structures cachées, les jeux sur le signifiant, la déconstruction de l'effet de réel, la parodie, les thématiques culturelles spécifiques comme l'horreur du nazisme et de la guerre,... etc, tous ces éléments ne sont repérables que si on les y a d'abord mis. Aussi n'est-il pas étonnant que la critique littéraire universitaire québécoise soit souvent obligée de se cantonner dans des approches formelles qui ont toujours un caractère répétitif lorsqu'au bout de l'analyse des narrateurs, narrataires et compagnie, on ne retrouve rien de bien original et de bien nouveau. La tentation est alors grande de se replier sur les études historiques et thématiques qui ont au moins l'avantage de faire progresser un certain type de connaissance sur les affaires d'ici, même quand l'idéologie derrière semble dépasser. La question de fond demeure; comment inventer un discours critique qui corresponde à notre société et aux œuvres québécoises? C'est peut-être par le biais d'une réflexion sur le tiersmondisme de notre littérature que nous pourrions délimiter une partie de réflexion 
théorique sur les notions de centre, de périphérie, d'écart ou encore d'une autre manière d'imitation qui inscrirait en même temps le littéraire et le théorique dans un intertexte culturel différent. La situation québécoise ne peut faire abstraction de sa propre genèse des formes littéraires, genèse qui s'inscrit mal dans la tradition européenne, à contre-courant, à contre-temps. Ici on doit tout inventer en même temps, le milieu comme le début et la fín. Enfin il semblerait important, essentiel, d'établir les bases d'un nouveau comparatisme entre les littératures d'Amérique dans la mesure où chacune d'elles a dû gagner son autonomie par rapport à la littérature-mère. Le cas des États-Unis devrait nous indiquer une partie de la voie, en ce sens qu'il est possible non seulement de gagner son autonomie mais encore d'exercer à rebours une influence. Nous n'en espérons pas autant. Mais tout cela suppose un certain décrochage, au moins une suspicion à l'égard de l'axe français de notre littérature. Il nous suffirait pour nous en convaincre d'examiner avec lucidité le regard que les Français portent eux-mêmes sur notre littérature et ce qu'ils en attendent. La réception de la littérature québécoise en France, maintenant mieux documentée ${ }^{6}$, permettrait de nous comprendre comme faisant partie de la littérature étrangère autant sinon plus que comme le petit cousin écrivain du cliché. Le comparatisme nord-sud aurait l'avantage de poser la question de notre appartenance à un même continent: voix identiques peut-être, langues différentes.

\section{La confusion des langues}

Il est impossible d'aborder la question de la littérature québécoise dans ses rapports à la langue française sans faire intervenir la tour de Babel. Celle de la légende mettait en scène une confusion des langues produites par la diversité. Nous parlerons de la confusion induite par la ressemblance. Prenons un exemple à la manière du Pierre Ménard de Borges. Supposons qu'un auteur québécois contemporain écrive la phase suivante: «Longtemps, je me suis couché de bonne heure. Parfois, à peine ma lumière éteinte, mes yeux se fermaient, si vite que je n'avais pas le temps de me dire: 'Je m'endors'. Et, une demi-heure après, la pensée qu'il était temps de chercher le sommeil m’éveillait; je voulais poser le volume que je croyais avoir encore dans les mains et éteindre ma lumière; je n'avais pas cessé en dormant de faire des réflexions sur ce que je venais de lire, mais ces réflexions avaient pris un tour un peu particulier; il me semblait que j'étais moi-même ce dont parlait l'ouvrage"n. On voit tout de suite la scène. Il s'agit évidemment d'un intellectuel. Les gens normaux se couchent après avoir regardé la télévision ou encore... L'intellectuel est celui qui a toujours besoin d'un livre pour s'endormir. Il se fait raconter des histoires comme le gamin qu'il n'a pas cessé d'être. Ou encore, il a oublié de prendre ses somnifères. C'est pour cela qu'il se réveille. Imagination maladive, il croit être ce qu'il lit. Va toujours, s'il s'agit d'un roman policier ou de l'un de ces best sellers dont on dévore les pages sans même les lire. Il n'y a là rien de bien spécial, tout le monde connaît cela: On imagine aussi la chambre dans une de ces vieilles maisons d'une cinquantaine d'années à Outremont, le lit "posturopédic», les oreillers bien calés sur la tête du lit, la douillette de duvet ou la courtepointe et la robe de chambre en velours côtelé. Parce que, quand je lis, c'est mon imagination qui s'empare des 
phrases, qui ramène les mots à ma langue maternelle, c'est-à-dire celle apprise à la maison, dans la rue, à l'école, dans les journaux, celle qui me donne une certaine emprise sur le réel "objectif». Mes notions d'espace et de temps sont inscrites dans ma langue; les images des mots sont les images de ma vie. La même phrase écrite par un Français prendrait un tout autre sens. On y verrait peut-être l'affirmation d'un moi, d'une conscience, un rapport qui s'installe entre la conscience et le temps qui s'écoule, un rapport à la littérature qui se fait dans la mémoire par delà les limites de la veille et du sommeil. Mais c'est parce que je connais l'autre langue, la pareille différente, que je peux faire cette hypothèse. Je sais traduire de l'une à l'autre, j'ai vécu dans les deux sociétés, je comprends un peu comment le transcodage peut se faire, sans être toujours sûr. Mais qu'en est-il de celui qui ne sait pas, de celui qui se permet d'avoir "des troubles avec son char", troubles que Furetière lui-même ne saurait dans quelle âme situer?

La pire confusion des langues est celle qui se produit du même au même, quand, comme dirait l'École, les mêmes signifiants ne renvoient pas aux mêmes signifiés. Cette situation insiste lourdement sur l'identité apparente de la langue et la disparité des cultures et des sociétés, comme si, soudainement, ces dernières n'avaient plus d'importance. Il est vrai que la différence linguistique postule automatiquement la différence culturelle et sociale. Nous n'imaginons pas une seule seconde en apprenant l'allemand que nous trouverons au-delà de la langue, une culture et une société identiques à celles de la langue de départ mais c'est qu'alors la langue est une frontière visible. Entre la France et le Québec, la langue est une frontière invisible que plusieurs écrivains ont voulu dévoiler en utilisant le dialecte, l'argot, en inventant le joual. Dans la perspective d'une prise en main de sa propre littérature, c'est une solution difficile, ponctuelle mais saine. N'en est-il pas ainsi de la langue américaine ou des langues latino-américaines? Serions-nous les seuls à jouir d'une sorte d'ubiquité culturelle et sociale? Il faut quitter Babel.

\section{La position du tiers}

Prendre un point de vue tiersmondiste sur notre littérature, c'est se décentrer par rapport à un certain nombre d'illusions savamment entretenues. Si nous avons réussi à créer une infrastructure complète sur le plan du livre, de son écriture et de sa diffusion, il nous manque un aspect important. Nous n'avons pas créé de société de lecteurs. Nous avons pris les lecteurs pour acquis, nous avons rassemblé dans un grand tout uniforme tous les lecteurs francophones d'ici et surtout d'ailleurs. Nous nous sommes adressés à la tête en oubliant le corps, ceux qui continuent à préférer les best-sellers, peu importe leur provenance, leur langue d'origine, ceux qui ont besoin de se faire raconter des histoires, de bonnes histoires. La préoccupation du lecteur d'ịci n'est pas évidente. Elle se développe mais on préfère mettre au compte de la mauvaise diffusion, ce qui est souvent vrai, les problèmes de la relation avec le lecteur. Il y a eu, il y a des succès de librairie au Québec parce que, quelque part, la magie s'établit entre l'écrivain et son public. Cela, si ce n'est pas du domaine de l'accident, n'a pu arriver que parce qu'on l'a voulu, on l'a cherché. 
Il est difficile de raconter l'histoire glorieuse du Québec qui n'a connu, de siècles en siècles depuis sa fondation, à peu près uniquement des défaites. Ça rend la pratique de l'épopée un tantinet difficile mais lorsqu'on lit Marquez par exemple, on se dit que ce qui n'est pas là peut toujours être inventé. Pourquoi ne pas s'inventer une histoire? Qu'est-ce qui importe, la vérité ou la littérature? Quand la scène politique et sociale est grise d'ennui, pourquoi ne pas inventer le rire? Le public du théâtre des Variétés sait peut-être mieux ce qu'est la vraie protestation... Pourquoi ne pas jouer à fond notre situation de tiers, situation de détachement au-delà des satisfactions d'être le tiers inclus du colonialisme ou le tiers exclu des révolutionnaires ou des contempteurs. Non, juste là, entre les deux: tiers, sans destin messianique, sans tragédie apocalyptique, petit pays heureux, heureux d'être industrialisé de force, riche par contiguité, à qui il est dévolu, qu'il le sache ou non, qu'il l'accepte ou non, de produire en ce moment du siècle, la seule et unique littérature franco-américaine que l'on est en droit d'attendre, conjointement avec les Antillais et tous ceux qui, quelque part sur l'axe nord-sud de l'Amérique, s'expriment dans leur français à eux et dont on reconnaîtra facilement les saveurs régionales. Vouloir être tiersmondiste, c'est aussi vouloir être nonaligné. C'est accepter de construire sa propre culture comme le mixte qu'elle doit être plutôt que d'attendre la reconnaissance ou de subir entre temps la construction des autres: inventer, de notre tiers à nous, une "littérature américaine directement écrite" ${ }^{7}$ en québécois.

1. Si ce texte était écrit dans la foulée de 1968 , je n'hésiterais pas à parler d'autocritique. Aujourd'hui, je préfère quelque chose comme "réflexion lucide et passionnée». Le «nous» est une tentative de collectivisation de cette réflexion. On comprendra alors que je ne sente pas le besoin, chemin faisant, de nommer des noms.

2. in Destin littéraire du québec, Montréal, Québec/Amérique, 1982, 208 p.

3. $f$ Gilles Marcotte, Je Roman à l'imparfait, Montréal, $\mathbf{H M H}, 1976,195$ p.

4. cf Gabrielle Poulin, Romans du pays 1968-1979, Montréal, Bellarmin 1980, 454 p.

5. cf André Belleau, le Romancier fictif, Québec, PUQ, 1980, 155 p.

6. $c f$ Lectures européennes de la littérature québécoise. Actes du Colloque international de Montréal (avril 1981), Montréal, Leméac, 1982,388 p.

Jacqueline Gerols, le Roman québécois en France, Montréal, HMH, 1984, 359 p., et Pierre Hébert, "La réception de la littérature canadienne-française en France, au XIX e sièclen, Voix et images, no 32, hiver 1986, p. 265 à 300.

7. La publicité du dernier roman de René Lapierre, l'Été Rebecca dit: Le roman d'un jeune Québécois qui est comme le signal d'une littérature américaine directement écrite en français.

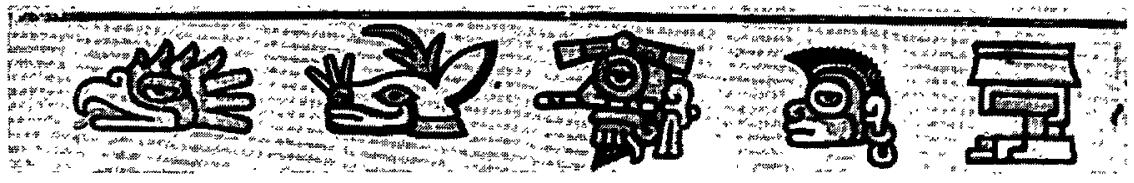

